\section{Adesão a listas de medicamentos essenciais em municípios de três estados brasileiros}

\author{
Adherence to essential medicines in cities from \\ three Brazilian states
}

\footnotetext{
${ }^{1}$ Faculdade de Farmácia, Universidade Federal do Rio Grande do Sul, Porto Alegre, Brasil.

2 Faculdade de Medicina,

Universidade Federal do Rio Grande do Sul, Porto Alegre, Brasil.

3 Centro de Ciências Biológicas e da Saúde, Universidade do Sul de Santa Catarina, Tubarão, Brasil.

4 Centro de Ciências da Saúde, Universidade de Caxias do Sul Caxias do Sul, Brasil.

5 Departamento de Medicina, Universidade da Região de Joinville, Joinville, Brasil. 6 Instituto de Ciências Básicas da Saúde, Universidade Federal do Rio Grande do Sul, Porto Alegre, Brasil.

7 Centro de Ciências Biológicas e da Saúde, Universidade Federal de Mato Grosso do Sul. Campo Grande, Brasil.

${ }^{8}$ Faculdade de Ciências

Biológicas e da Saúde, Centro

Universitário da Grande

Dourados, Dourados, Brasil.

Correspondência

T. S. Dal Pizzol

Faculdade de Farmácia

Universidade Federal do Rio

Grande do Sul.

Av. Ipiranga 2752, 2o andar

Porto Alegre, RS

90610-000, Brasil.

tatiane.silva@ufrgs.br
}

Tatiane da Silva Dal Pizzol 1,2

Daisson José Trevisol 3

Isabela Heineck 1

Liziane Maahs Flores 2,4

Aline Lins Camargo 4

Álvaro Köenig 5

Iraci Lucena da Silva Torres 6

Mônica Cristina Toffoli Kadri 7

Maria Tereza Ferreira Duenhas Monreal 7

Adriana Mary Mestriner Felipe de Melo 8

Maria Beatriz Cardoso Ferreira 6

\section{Introdução}

This study compared the Municipal Essential Medicines Lists (REMUME) and examined adherence by prescribers and availability of essential medicines in the health units affiliated with the Unified National Health System (SUS). Data were collected on lists and medicines prescribed to 2,411 patients enrolled consecutively in primary or secondary care services in Brazilian municipalities. Of 5,222 prescribed medicines, $76.4 \%$ were present on the REMUME, $76.8 \%$ on the National List of Essential Medicines (RENAME), and $63 \%$ on the World Health Organization (WHO) list. Among the most frequently prescribed medications, one or more did not belong to the respective REMUME. Of all medicines prescribed, 76.1\% were available in the inspected facilities; for essential medicines, the availability increased to $88.1 \%$. Prescription in disagreement with the REMUME may result from the unavailability of medicines in the inspected facilities or the lists' inadequacy for the level of care.

Essential Drugs; National Drug Policy; Single Health System
Medicamentos essenciais, de acordo com a Organização Mundial da Saúde (OMS), são aqueles que satisfazem às necessidades de saúde prioritárias da população, os quais devem ser selecionados por critérios de eficácia, segurança, conveniência, qualidade e custo favorável. Eles devem estar acessíveis em todos os momentos, na dose apropriada, a todos os segmentos da sociedade 1. Em meio à multiplicidade de medicamentos disponíveis no mercado e aos avanços técnico-científicos que levam ao desenvolvimento de novos fármacos, o conceito de medicamentos essenciais vem sendo aceito e adotado em diversas partes do mundo, amparado pelo paradigma da prática clínica baseada em evidências e pela necessidade de ampliar o acesso a um maior contingente de pessoas.

O Sistema Único de Saúde (SUS) é constituído pelo sistema de saúde público e suplementar, estabelecido pela Constituição Federal em 1988 e pela Lei Orgânica da Saúde 2 , em 1990. De acordo com os princípios do SUS, é garantido o acesso universal e igualitário aos serviços de saúde, incluindo-se medicamentos, previsto em 1990, mas regulamentado apenas em 1998, quando a Política Nacional de Medicamentos foi publicada. Este documento tem como propósito garantir a eficácia, segurança e qualidade dos medicamentos, a promoção do uso racional e o acesso universal àqueles considerados essenciais ${ }^{3}$. 
O Brasil elaborou em 1964 sua primeira relação básica de medicamentos considerados essenciais, antes da recomendação da OMS, em 19774. Apesar desse pioneirismo, a descontinuidade das políticas públicas nas décadas subseqüentes e os longos períodos em que a Relação Nacional de Medicamentos Essenciais (RENAME) ficou sem revisão podem ter atrasado o processo de consolidação do conceito de medicamento essencial entre gestores, profissionais de saúde e usuários 5 .

Passados cerca de trinta anos após a introdução do conceito de medicamento essencial pela OMS, faz-se necessário avaliar se os programas de medicamentos essenciais foram de fato implementados e se produziram impacto na racionalização da terapêutica, como a eliminação de medicamentos ineficazes das listas nacionais e locais 6 . Os estudos nacionais sobre o tema têm enfocado principalmente a prescrição de medicamentos constantes na RENAME ou nas Relações Municipais de Medicamentos Essenciais (REMUME), sendo que estas últimas devem ser elaboradas para atender às necessidades específicas da população de cada município, tendo a RENAME como modelo. Aspectos referentes à elaboração, às características das REMUME e à disponibilidade destes medicamentos têm sido pouco investigados, tanto no Brasil quanto em outras nações que adotaram políticas de medicamentos essenciais $5,7,8$. Entre as formas de medir a adesão aos programas de medicamentos essenciais, o percentual de medicamentos prescritos incluídos nas REMUME tem sido utilizado como proxy da adesão.

Uma das formas de medir a adesão a esta política é pela prescrição de medicamentos incluídos na REMUME.

Nesse sentido, foi desenvolvido estudo multicêntrico com o objetivo de verificar a adesão às listas de medicamentos essenciais e a disponibilidade dos mesmos em unidades de saúde de oito municípios brasileiros.

\section{Métodos}

A presente análise faz parte de estudo multicêntrico, com delineamento transversal, envolvendo oito municípios de três estados brasileiros (Rio Grande do Sul, Santa Catarina e Mato Grosso do Sul).

\section{Os municípios e unidades investigadas}

As principais características dos municípios participantes da pesquisa e do serviço de saúde onde os dados foram coletados são apresentadas na
Tabela 1. Os municípios podem ser agrupados de acordo com o número de habitantes, em: menos de 100 mil habitantes [Lajeado (Rio Grande do Sul) e Tubarão (Santa Catarina)], entre $100 \mathrm{mil}$ e 200 mil habitantes [Passo Fundo (Rio Grande do Sul) e Dourados (Mato Grosso do Sul)], entre 300 mil e 500 mil habitantes [Caxias do Sul (Rio Grande do Sul) e Joinville (Santa Catarina)] e com mais de 500 mil habitantes [Campo Grande (Mato Grosso do Sul) e Porto Alegre (Rio Grande do Sul)]. Com exceção de Passo Fundo, os demais municípios apresentam índices de desenvolvimento humano (IDH) acima dos índices dos respectivos estados (Mato Grosso do Sul - 0,778, Rio Grande do Sul - 0,814, Santa Catarina - 0,822). Apenas um município (Dourados) apresenta IDH inferior ao da média nacional: 0,800.

Todos os serviços avaliados apresentavam nível de atendimento secundário; destes, seis apresentavam nível de atenção primária.

\section{Descrição dos locais de pesquisa}

Fizeram parte do estudo oito unidades básicas de saúde (UBS) ou unidades de atendimento ambulatorial vinculadas e/ou conveniadas às instituições universitárias participantes da pesquisa, com níveis de atenção ambulatorial primária, secundária e terciária. Os critérios de inclusão das unidades foram: (1) unidade vinculada a universidades parceiras da Universidade Federal do Rio Grande do Sul (UFRGS); (2) unidade localizada em municípios de diferentes portes, categorizados de acordo com o número de habitantes; (3) unidade que oferecesse estágios para estudantes de graduação e/ou pós-graduação da área da saúde (incluindo aqueles vinculados à residência médica), os quais teriam contato com diferentes padrões de prescrição.

\section{Amostragem}

Para cada um dos 12 meses do ano, foi realizado sorteio de uma semana do mês, de três dias nesta semana e de um turno específico em cada dia sorteado. Para a realização da coleta dos dados foi considerado como turno da manhã o período compreendido entre as $7 \mathrm{~h}$ e $12 \mathrm{~h}$, e, como turno da tarde, aquele compreendido entre $12 \mathrm{~h}$ e $17 \mathrm{~h}$.

A amostra foi formada a partir da prescrição dos pacientes presentes no dia e no turno sorteados para a coleta de dados. Foram arrolados consecutivamente, por conveniência, oito pacientes por dia de pesquisa. Caso não fossem obtidos oito pacientes por dia, realizava-se coleta em mais um dia da semana. Houve preocupação em entrevistar no mínimo 24 pacientes por mês em cada local, o que trouxe, por conseqüência, 
Características dos municípios, serviços de saúde e Relações Municipais de Medicamentos Essenciais (REMUME) avaliados, agrupados por número de habitantes.

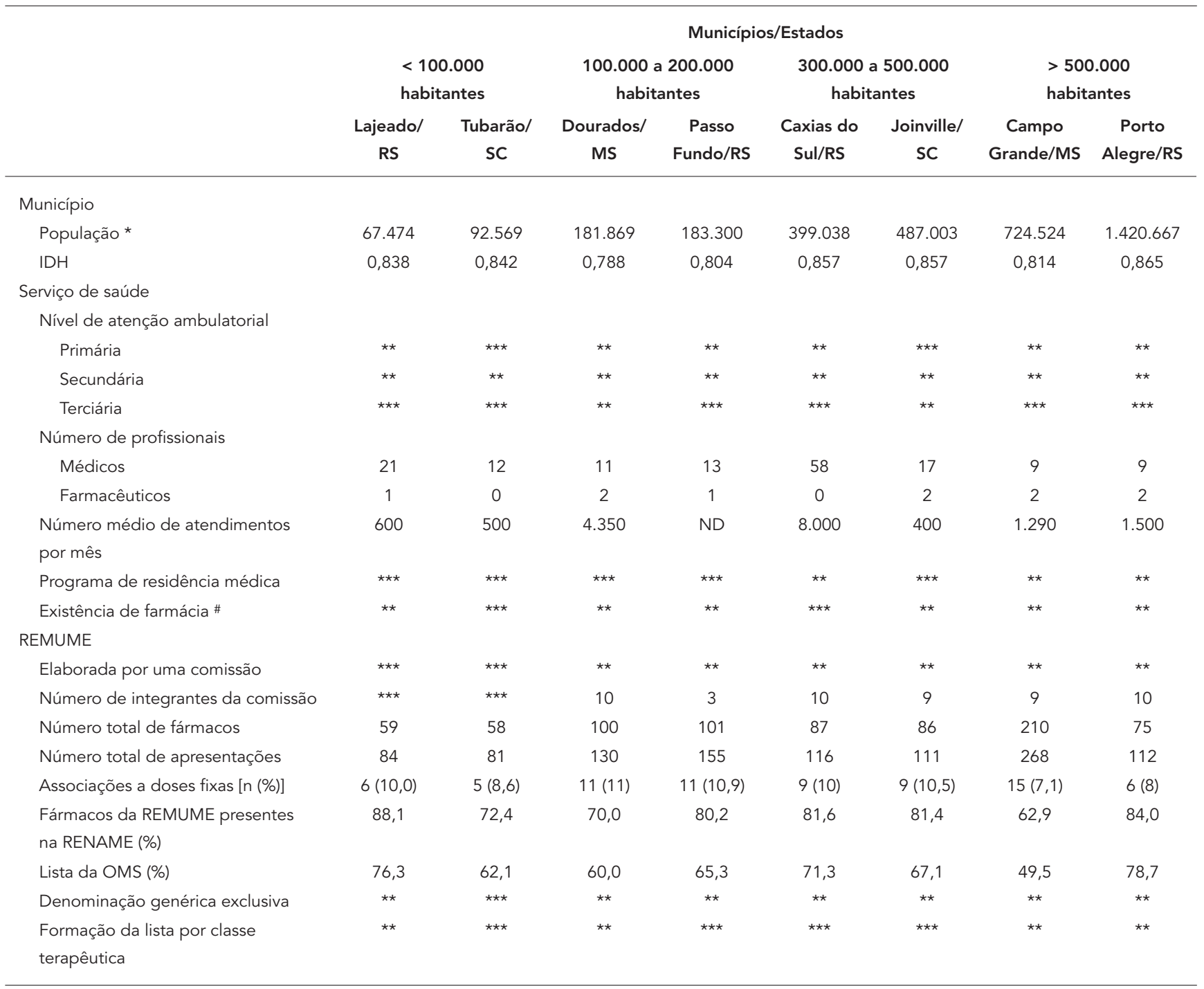

IDH: índice de desenvolvimento humano; ND: dado não disponível; OMS: Organização Mundial da Saúde; RENAME: Relação Nacional de Medicamentos Essenciais.

Estados: MS: Mato Grosso do Sul; RS: Rio Grande do Sul; SC: Santa Catarina.

* Dados do Instituto Brasileiro de Geografia e Estatística (http://www.ibge.gov.br);

** Apresenta a característica;

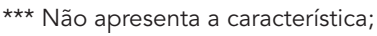

\# Farmácia junto ao serviço de saúde no qual foi realizada a consulta geradora da receita médica.

um número amostral $4 \%$ maior do que o estimado (que era de 288 prescrições por município estudado).

Após a consulta médica, foi preenchida uma ficha de coleta de dados específica, para cada paciente portador de receita médica incluído na amostra. Os pacientes eram abordados na saída dos consultórios ou da farmácia.
Foram incluídas as prescrições de pacientes com idade igual ou superior a 18 anos que aceitaram fazer parte da pesquisa.

\section{Coleta de dados}

Informações sobre os municípios foram obtidas nas páginas eletrônicas do Instituto Brasi- 
leiro de Geografia e Estatística (IBGE. Cidades@. http://www.ibge.gov.br/cidadesat/default.php) e Programa das Nações Unidas para o Desenvolvimento (PNUD-Brasil. http://www.pnud.org. br/idh/). Informações sobre os serviços de saúde foram obtidas com as coordenações dos locais pesquisados.

Uma ficha montada especificamente para o estudo foi preenchida pelos coletadores a partir das prescrições dos pacientes. Os seguintes dados foram coletados: nome do medicamento prescrito, forma farmacêutica, concentração/ dose, via e intervalo de administração, duração de tratamento e disponibilidade dos medicamentos prescritos na farmácia, no momento da coleta dos dados. A partir desses dados, foi avaliado se os medicamentos prescritos constavam da REMUME de cada um dos oito municípios pesquisados, da RENAME 9 e da 14a Lista Modelo de Medicamentos Essenciais 1 da OMS. As edições selecionadas da RENAME e da lista da OMS correspondiam àquelas em vigência na época em que foi iniciada a coleta dos dados. Foram considerados "medicamentos não-essenciais" aqueles que não pertenciam às listas vigentes na época da coleta dos dados. A adesão às listas pelos profissionais prescritores foi avaliada pela prescrição de medicamentos incluídos nas listas.

As REMUME foram comparadas entre si, com a RENAME e com a lista da OMS em relação aos itens: número de fármacos e apresentações, presença dos medicamentos das REMUME na RENAME e na lista da OMS, presença de associações a doses fixas e forma de apresentação das listas. Não foram contabilizados componentes das listas definidos pela RENAME como outros produtos essenciais (desinfetantes, hemoderivados, nutrição e reposição hidroeletrolítica parenteral, solução para diálise, vacinas, soros e imunoglobulinas), tendo em vista que esses produtos não são normalmente prescritos ao paciente para uso domiciliar.

Os investigadores buscaram, junto aos locais onde a coleta foi feita ou nas secretarias municipais de saúde, dados sobre a elaboração das REMUME, incluindo comissão responsável, critérios para elaboração e atualização.

A disponibilidade dos medicamentos prescritos, definida neste estudo como a disponibilidade física na farmácia nos dias em que a coleta foi realizada, foi calculada pela divisão do número de medicamentos disponíveis pelo número de medicamentos prescritos para cada paciente, e o resultado expresso em percentual.

Se o medicamento estava presente na REMUME e era fornecido pela farmácia, mas não havia unidades em estoque disponível ao paciente no dia da consulta médica, classificavase o medicamento como não disponível.

\section{Processamento e análise dos dados}

Os dados foram reunidos e codificados em banco de dados por meio do programa Epi Data, versão 1.5 (Epidata Assoc., Odense, Dinamarca). A análise estatística foi realizada por meio do programa SPSS versão 16.0 for Windows (SPSS Inc., Chicago, Estados Unidos). Os resultados foram expressos como freqüência absoluta, freqüência relativa, média e desvio-padrão (DP). A unidade de análise foi o medicamento prescrito aos pacientes entrevistados.

\section{Aspectos éticos}

O projeto foi submetido e aprovado pelos Comitês de Ética e Pesquisa das instituições envolvidas. O termo de consentimento informado foi lido e assinado pelo entrevistado e pelo entrevistador. $\mathrm{O}$ estudo não interferiu com o tratamento proposto ou com as prescrições medicamentosas e não-medicamentosas recebidas pelos pacientes, respeitando-se as determinações estabelecidas pelos profissionais da área de saúde envolvidos no seu atendimento.

\section{Resultados}

Características das REMUME e dos locais investigados

A Tabela 1 apresenta características das REMUME, dos serviços de saúde e dos municípios onde a pesquisa foi realizada. O processo de elaboração das REMUME foi conduzido por uma comissão em seis municípios participantes dessa análise. Nos outros dois municípios, a elaboração da REMUME foi realizada por um farmacêutico.

Todas as comissões eram compostas por médicos (variando de 1 a 7 profissionais) e farmacêuticos (1 a 5). Quatro comissões possuíam enfermeiro ( 1 a 3 ), três possuíam um administrador e duas um dentista. Em uma comissão havia representantes dos setores de compras, financeiro e jurídico.

Em relação à última atualização das REMUME, duas foram atualizadas em 2008, uma em 2007, três em 2006 e duas em 2004. Dados sobre os critérios utilizados para a elaboração das REMUME não foram disponibilizados em três municípios. Nos demais, foram mencionados critérios como prevalência da doença, eficácia, segurança, custo, presença do medicamento na 
RENAME ou na relação estadual e solicitação dos médicos.

Denominação genérica foi utilizada exclusivamente em sete listas, e, em uma, alguns medicamentos foram listados somente pelo nome comercial. As REMUME analisadas apresentavam, em média, 97 ( \pm 48,5) fármacos e $132( \pm 59,8)$ apresentações. O percentual de associações a doses fixas foi, em média, de 9,5\% ( $\pm 1,4 \%$ ), superior ao da RENAME (9\%) e inferior ao da lista da OMS (12,2\%).

Foram encontrados 476 medicamentos diferentes nas receitas avaliadas, prescritos 5.222 vezes a 2.411 pacientes, correspondendo a 2,17 $(1,49)$ medicamentos por prescrição. Observouse $0,5 \%$ de medicamentos não identificados, em decorrência de letras ilegíveis ou ausência de registro na Agência Nacional de Vigilância Sanitária (ANVISA).

Do total de medicamentos prescritos nos locais estudados, $76,4 \%$ constavam nas REMUME, $76,8 \%$ na RENAME e 63\% na lista da OMS (Tabela 2). A variação entre os locais investigados foi de $25,7 \%$ a $92,9 \%$ para a REMUME, $45,4 \%$ a $88,2 \%$ para a RENAME e $34,9 \%$ a $72,1 \%$ para a lista da OMS.

Também foram identificados os dez medicamentos mais prescritos em cada unidade de saúde e sua presença na respectiva REMUME. Entre os dez mais prescritos de cinco municípios, um ou mais não pertenciam às respectivas REMUME: dipirona (Caxias do Sul), enalapril (Lajeado e Porto Alegre), sinvastatina (Lajeado e Tubarão), fluoxetina (Porto Alegre), diosmina (Joinvile), budesonida (Joinvile), omeprazol (Tubarão) e betametasona (Tubarão).

\section{Disponibilidade dos medicamentos prescritos}

Do total de medicamentos prescritos, $76,1 \%$ estavam disponíveis nas farmácias dos locais pesquisados nos dias em que a coleta de dados foi realizada, variando de $51 \%$ a $93 \%$ (Tabela 3). Quando analisados os medicamentos essenciais em separado, a disponibilidade aumentou em todos os locais, com média geral de $88,1 \%$. Dois locais foram excluídos dessa análise por não possuírem farmácia na unidade. A Tabela 3 mostra também os dez medicamentos menos disponíveis em cada local, no período analisado.

\section{Discussão}

O presente estudo apresenta características das listas de medicamentos essenciais e avalia aspectos da adesão às listas em oito unidades de saúde de três estados brasileiros, a partir das prescri- ções médicas e da disponibilidade dos medicamentos nos locais investigados.

A presença de 76,8\% dos medicamentos prescritos na RENAME foi semelhante à encontrada em pesquisa nacional envolvendo 29 unidades de saúde pertencentes a cinco capitais brasileiras e outros cinco municípios distantes dos grandes centros e com rede de serviços de menor complexidade $(78,3 \%) 10$. O percentual de medicamentos prescritos presentes nas REMUME apresentou variações importantes entre os municípios (25,7\% a 92,9\%), com percentual médio de $76,4 \%$. Em estudos anteriores, os percentuais variaram de $58,4 \%$ a $92,7 \% 11,12,13,14,15,16,17$. O percentual verificado também é inferior aos observados em países da África, que variaram entre 88\% e 92,3\% 18,19,20,21 e Ásia, com 85\% a 99,7\% 18,22,23,24. Na avaliação desses dados, não podemos descartar a possibilidade de que a amostra selecionada tenha superestimado o percentual de medicamentos essenciais prescritos. Como a coleta foi realizada na saída das farmácias, na maioria dos locais, usuários previamente informados sobre a não disponibilidade dos medicamentos nas farmácias (por não fazer parte da lista ou por desabastecimento) não passavam por lá, perdendo a chance de pertencer à amostra.

Considerando que a seleção dos medicamentos deve observar aspectos epidemiológi$\cos 4$, era de se esperar maior concordância das prescrições com as REMUME, em seguida com a RENAME e menor concordância com a lista da OMS. Nos municípios do Mato Grosso do Sul e em Passo Fundo, essa ordem foi observada. Fator que pode estar determinando maior adequação da prescrição em relação a esse aspecto é o número de medicamentos presentes nas REMUME dos municípios citados. Estas possuíam o maior número de medicamentos e apresentações, dentre as REMUME analisadas. Outros fatores que podem ter contribuído para o percentual elevado são a existência de uma comissão multidisciplinar responsável pela elaboração da REMUME, a adoção de critérios para a sua elaboração e a existência de farmacêuticos na equipe dos serviços onde a pesquisa foi realizada.

Os menores percentuais de adesão às listas foram verificados nos municípios com menos de 100 mil habitantes e naqueles com 300 mil a 500 mil habitantes investigados. Nos municípios com 300 mil a 500 mil habitantes investigados, a baixa adesão à REMUME pode estar relacionada ao nível de complexidade da atenção. Em um deles, o nível de atendimento era predominantemente secundário, com ambulatório de referência para diversas especialidades, tais como cirurgia bucofacial, urologia, dermatologia, oftalmologia, hematologia e infectologia, entre outras. Além dis- 
Presença dos medicamentos prescritos nas Relações Municipais de Medicamentos Essenciais (REMUME), Relação Nacional de Medicamentos Essenciais (RENAME) 9 e 14ạ Lista Modelo de Medicamentos Essenciais 1 da Organização Mundial da Saúde (OMS).

\begin{tabular}{|c|c|c|c|c|c|c|}
\hline \multirow{3}{*}{$\begin{array}{l}\text { Municípios/Estados (número de } \\
\text { medicamentos prescritos) }\end{array}$} & \multicolumn{6}{|c|}{ Presença dos medicamentos prescritos nas listas } \\
\hline & \multicolumn{2}{|c|}{ REMUME } & \multicolumn{2}{|c|}{ RENAME } & \multicolumn{2}{|c|}{ Lista da OMS } \\
\hline & $\mathrm{n}$ & $\%$ & $\mathrm{n}$ & $\%$ & $\mathrm{n}$ & $\%$ \\
\hline \multicolumn{7}{|l|}{$<100.000$ habitantes } \\
\hline Lajeado/RS (713) & 509 & 71,4 & 611 & 85,7 & 489 & 68,6 \\
\hline Tubarão/SC (152) & 39 & 25,7 & 69 & 45,4 & 53 & 34,9 \\
\hline \multicolumn{7}{|l|}{100.000 a 200.000 habitantes } \\
\hline Dourados/MS (604) & 561 & 92,9 & 506 & 83,8 & 324 & 53,6 \\
\hline Passo Fundo/RS (824) & 752 & 91,3 & 701 & 85,1 & 541 & 65,7 \\
\hline \multicolumn{7}{|l|}{300.000 a 500.000 habitantes } \\
\hline Caxias do Sul/RS (893) & 463 & 51,8 & 539 & 60,4 & 469 & 52,5 \\
\hline Joinville/SC (578) & 361 & 62,5 & 315 & 54,5 & 408 & 70,6 \\
\hline \multicolumn{7}{|l|}{$>500.000$ habitantes } \\
\hline Campo Grande/MS (837) & 777 & 92,8 & 738 & 88,2 & 553 & 66,5 \\
\hline Porto Alegre/RS (621) & 525 & 84,5 & 531 & 85,5 & 447 & 72,1 \\
\hline Total (5.222) & 3.987 & 76,4 & 4.010 & 76,8 & 3.284 & 63,0 \\
\hline
\end{tabular}

Estados: MS: Mato Grosso do Sul; RS: Rio Grande do Sul; SC: Santa Catarina.

so, desconhecimento da lista de medicamentos essenciais, mesmo em locais onde há programas de residência, pode contribuir para uma menor adesão. Estudo sobre a influência da propaganda de medicamentos em uma escola de medicina do Sul do Brasil revelou que a maioria dos estudantes de medicina desconhecia a RENAME ou a lista da OMS 25.

Algumas características dos municípios com menos de 100 mil habitantes, tais como inexistência de comissão responsável pela elaboração da REMUME e inexistência de farmácia vinculada ao serviço de saúde em um deles ou elevado percentual de "medicamentos não-essenciais" disponíveis na farmácia de outro, sugerem dificuldades no desenvolvimento e na implementação da assistência farmacêutica nestes municípios. A adesão dos prescritores à REMUME pressupõe um programa de medicamentos essenciais inserido em uma política de assistência farmacêutica municipal eficiente 26,27,28.

Chama a atenção que nos dois municípios com menor percentual de adesão à REMUME, não havia farmácia vinculada à unidade, sugerindo que a dispensação de medicamentos na própria unidade de atendimento é fator importante para a adesão à REMUME 28 .

Na presente análise, não foram investigadas as causas individuais para a prescrição dos me- dicamentos que não fazem parte das REMUME, a partir de condições específicas do profissional, do paciente e do serviço de saúde. No entanto, algumas hipóteses podem ser levantadas, considerando as alternativas disponíveis em cada REMUME e as evidências disponíveis sobre a essencialidade do medicamento em questão. Em alguns casos, a prescrição do "medicamento não-essencial” pode ser justificada pela inadequação da lista, ao apresentar lacunas terapêuticas 16. São exemplos a ausência da sinvastatina nas REMUME de dois municípios, e da budesonida na REMUME de outro. Nesses casos, nenhum hipolipemiante ou corticóide inalatório para tratamento da asma brônquica estava presente nas listas dos respectivos municípios.

Entretanto, na maioria dos casos, a prescrição do medicamento não essencial parece estar relacionada à restrição imposta pelas listas, que propunham um único representante para algumas categorias terapêuticas. A prescrição de enalapril, em vez de captopril, e de dipirona, em vez de paracetamol ou ibuprofeno, são exemplos em que não fica claro se o medicamento não essencial foi prescrito devido à falta de adesão do prescritor à REMUME, às características epidemiológicas da população assistida ou às condições particulares dos pacientes. 
Tabela 3

Disponibilidade de medicamentos essenciais e "não-essenciais" nas farmácias dos locais investigados $(n=4.119)$ *

\begin{tabular}{|c|c|c|c|c|c|c|c|}
\hline \multirow[t]{2}{*}{ Municípios/Estados } & \multicolumn{2}{|c|}{$\begin{array}{l}\text { Medicamentos } \\
\text { disponíveis }\end{array}$} & \multicolumn{2}{|c|}{$\begin{array}{l}\text { Medicamentos } \\
\text { essenciais } \\
\text { disponíveis ** }\end{array}$} & \multicolumn{2}{|c|}{$\begin{array}{l}\text { "Medicamentos } \\
\text { não-essenciais" } \\
\text { disponíveis ** }\end{array}$} & \multirow[t]{2}{*}{$\begin{array}{l}\text { Principais medicamentos } \\
\text { não disponíveis }\end{array}$} \\
\hline & $\mathrm{n} /$ Total & $\%$ & $\mathrm{n} /$ Total & $\%$ & $\mathrm{n} /$ Total & $\%$ & \\
\hline Campo Grande/MS & $778 / 837$ & 93,0 & 777/777 & 100,0 & $1 / 60$ & 1,7 & $\begin{array}{l}\text { Sinvastatina ***, } \\
\text { escopolamina ***, captopril, ácido } \\
\text { acetilsalicílico, hidroclorotiazida, } \\
\text { furosemida, atenolol }{ }^{* \star *} \text {, diclofenaco, } \\
\text { parafina líquida ***, metformina }\end{array}$ \\
\hline Dourados/MS & $539 / 604$ & 89,2 & $539 / 561$ & 96,1 & $0 / 40$ & 0 & $\begin{array}{l}\text { Captopril, hidroclorotiazida, } \\
\text { diclofenaco, fluoxetina, } \\
\text { fenilbutazona } * \star \star \text {, dipirona, amitriptilina, } \\
\text { metformina, glibenclamida, fluconazol }\end{array}$ \\
\hline Lajeado/SC & $504 / 713$ & 70,7 & $449 / 509$ & 88,2 & $54 / 199$ & 27,1 & $\begin{array}{c}\text { Hidroclorotiazida, ácido acetilsalicílico, } \\
\text { enalapril ***, paracetamol, omeprazol, } \\
\text { captopril, sinvastatina }{ }^{\star \star \star} \text {, ibuprofeno, } \\
\text { glibenclamida, digoxina }\end{array}$ \\
\hline Passo Fundo/RS & $606 / 766$ & 79,1 & $598 / 701$ & 85,3 & $8 / 65$ & 12,3 & $\begin{array}{l}\text { Captopril, ácido acetilsalicílico, } \\
\text { hidroclorotiazida, paracetamol, } \\
\text { ranitidina, ibuprofeno, sinvastatina, } \\
\text { metformina, dexclorfeniramina, } \\
\text { propranolol }\end{array}$ \\
\hline Porto Alegre/RS & $317 / 621$ & 51,0 & $306 / 525$ & 58,3 & $11 / 96$ & 11,4 & 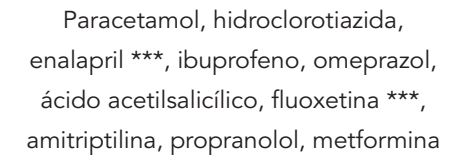 \\
\hline Joinville/SC & $392 / 578$ & 67,8 & $355 / 361$ & 98,3 & $37 / 217$ & 17,0 & $\begin{array}{l}\text { Diosmina ***, outros produtos (ATC: } \\
\text { V03AX), inibidores da agregação } \\
\text { plaquetária, excluindo heparina (ATC: } \\
\text { BO1AC), varfarina, amitriptilina, } \\
\text { fluoxetina, budesonida ***, furosemida, } \\
\text { carbamazepina }\end{array}$ \\
\hline Total & $3.136 / 4.119$ & 76,1 & $3.024 / 3.434$ & 88,1 & $111 / 677$ & 16,4 & $\begin{array}{c}\text { Hidroclorotiazida, captopril, } \\
\text { paracetamol, ácido acetilsalicílico, } \\
\text { diosmina, ibuprofeno, omeprazol, } \\
\text { sinvastatina, enalapril, outros produtos } \\
\text { (ATC: V03AX) }\end{array}$ \\
\hline
\end{tabular}

ATC: Anatomical Therapeutic Chemical Classification 30; REMUME: Relação Municipal de Medicamentos Essenciais. Estados: MS: Mato Grosso do Sul; RS: Rio Grande do Sul; SC: Santa Catarina.

* Excluídos Caxias do Sul e Tubarão por não possuírem farmácias nos locais onde a coleta dos dados foi realizada;

** De acordo com a REMUME;

*** Não pertence à REMUME do respectivo município.

Em que pese a complexidade e dificuldade de medir acesso a medicamentos 10 , poucos estudos realizados no Brasil investigaram aspectos relacionados ao acesso a medicamentos essenciais em unidades públicas de saúde. Entre os elementos que compõem o acesso, indicadores de disponibilidade dos medicamentos nos ser- viços têm sido investigados, com variações conceituais entre os pesquisadores. Para análise da disponibilidade de medicamentos-chave, Naves \& Silver $14 \mathrm{e}$ Cunha et al. 12 utilizaram uma lista de quarenta medicamentos considerados marcadores, conforme metodologia proposta pela OMS. Em outro estudo de abrangência nacional, 
foi verificada disponibilidade de $73 \%$ para uma lista de 15 medicamentos considerados principais, além de contraceptivo oral e preservativo masculino, em 29 unidades de saúde, pertencentes a cinco capitais brasileiras e outros cinco municípios menores 10 . Nesses estudos, o indicador de disponibilidade foi calculado pela divisão do número de medicamentos marcadores disponíveis na unidade pelo número total de medicamentos marcadores da lista, conforme proposto pela OMS ${ }^{29}$. Assim, a comparação com esses dados deve ser feita com cautela, já que, no presente estudo, foi avaliada a disponibilidade pontual para todos os medicamentos prescritos, na própria unidade de saúde onde a pesquisa foi feita e no mesmo dia da entrevista do paciente.

\section{Conclusões}

Avaliação da adesão às listas de medicamentos essenciais municipais é importante indicador de qualidade das prescrições, uma vez que estas listas são concebidas a partir da epidemiologia das doenças e de critérios de eficácia, segurança e qualidade dos medicamentos. A ausência de aproximadamente $24 \%$ dos medicamentos pres- critos nas listas municipais pode ser resultado da não-adesão dos prescritores aos padrões de seleção empregados na elaboração dessas listas e do não-conhecimento da lista de medicamentos essenciais disponíveis no município. Por outro lado, esse resultado pode indicar que as REMUME analisadas não estão contemplando adequadamente as necessidades terapêuticas dos serviços de saúde investigados. Deve-se salientar que as REMUME analisadas foram elaboradas para atender às necessidades de saúde no âmbito da atenção primária e que alguns dos centros investigados apresentaram níveis de atenção secundária e terciária. O desabastecimento de medicamentos também pode ter contribuído para que o prescritor optasse pela prescrição de um "medicamento não-essencial". Outros aspectos relacionados com a assistência farmacêutica municipal, como existência de farmácia na unidade de saúde, podem influenciar no sucesso de um programa de medicamentos essenciais. Para aumentar a adesão à REMUME, é necessário que a atualização periódica do elenco de medicamentos, realizada por uma comissão multidisciplinar, caminhe junto com a divulgação da lista aos prescritores e com o abastecimento regular dos medicamentos nas farmácias.

\section{Resumo}

Este estudo comparou as Relações Municipais de Medicamentos Essenciais (REMUME), analisou a adesão dos prescritores e a disponibilidade de medicamentos essenciais em unidades de saúde vinculadas ao Sistema Único de Saúde (SUS). Foram coletados dados sobre as REMUME e os medicamentos prescritos a 2.411 pacientes arrolados consecutivamente em oito serviços de atenção primária ou secundária de municípios da região centro-sul do país. A disponibilidade dos medicamentos foi verificada por meio da existência de estoque na farmácia. De um total de 5.222 medicamentos prescritos, 76,4\% constavam nas REMUME, 76,8\% na Relação Nacional de Medicamentos Essenciais
(RENAME) e 63\% na lista da Organização Mundial da Saúde (OMS). Entre os medicamentos mais prescritos, um ou mais não pertenciam às respectivas REMUME. Do total de medicamentos prescritos, 76,1\% estavam disponíveis nos locais pesquisados; entre os medicamentos essenciais, a disponibilidade aumentou para 88,1\%. A prescrição em desacordo com as REMUME pode ser resultado da indisponibilidade dos medicamentos nos locais investigados ou da inadequação das listas frente ao nível de complexidade da atenção.

Medicamentos Essenciais; Política Nacional de Medicamentos; Sistema Único de Saúde 


\section{Colaboradores}

T. S. Dal Pizzol participou da concepção do estudo, coordenação da pesquisa em Passo Fundo, redação e revisão crítica do manuscrito. A. M. M. F. Melo participou da concepção do estudo, coordenação da pesquisa em Dourados e revisão crítica do manuscrito. M. T. F. D. Monreal participou da concepção do estudo, coordenação da pesquisa em Campo Grande, e revisão crítica do manuscrito. M. C. T. Kadri colaborou na concepção do estudo e revisão crítica do manuscrito. A. Köenig participou da concepção do estudo, coordenação da pesquisa em Joinville e revisão crítica do manuscrito. I. L. S. Torres participou da concepção do estudo, coordenação da pesquisa em Lajeado e revisão crítica do manuscrito. A. L. Camargo participou da concepção do estudo, coordenação da pesquisa em Caxias do Sul, redação e revisão crítica do manuscrito. L. M. Flores participou da concepção do estudo, coordenação da pesquisa em Porto Alegre, redação e revisão crítica do manuscrito. I. Heineck contribuiu na concepção do estudo, redação e revisão crítica do manuscrito. D. J. Trevisol participou da concepção do estudo, coordenação da pesquisa em Tubarão, redação e revisão crítica do manuscrito. M. B. C. Ferreira contribuiu na concepção do estudo, coordenação geral da pesquisa e revisão crítica do manuscrito.

\section{Agradecimentos}

Ao Conselho Nacional de Desenvolvimento Científico e Tecnológico (CNPq), pelo auxílio financeiro, à Fundação de Amparo à Pesquisa do Estado do Rio Grande do Sul (FAPERGS) e Pró-Reitoria de Pesquisa da Universidade Federal do Rio Grande do Sul (PROPESQ/UFRGS), pela disponibilidade de bolsas de iniciação científica.

\section{Referências}

1. World Health Organization. WHO model list. 14th Ed. Geneva: World Health Organization; 2005.

2. Brasil. Lei no. 8.080, de 19 de setembro de 1990. Dispõe sobre as condições para a promoção, proteção e recuperação da saúde, a organização e o funcionamento dos serviços correspondentes e dá outras providências. Diário Oficial da União 1990; 20 set.

3. Vieira FS, Zucchi P. Distorções causadas pelas ações judiciais à política de medicamentos no Brasil. Rev Saúde Pública 2007; 41:214-22.

4. Gerência Técnica de Assistência Farmacêutica, Departamento de Atenção Básica, Secretaria de Políticas de Saúde, Ministério da Saúde. Relação nacional de medicamentos essenciais - RENAME. 4a Ed. Brasília: Ministério da Saúde; 2007.
5. Guerra Jr. AA, Acúrcio FA, Gomes CA, Miralles M, Girardi SN, Werneck GA, et al. Availability of essential drugs in two regions of Minas Gerais, Brazil. Rev Panam Salud Pública 2004; 15:168-75.

6. Levy M, Reidenberg MM. What has been the impact of the concept of essential drugs? Clin Pharmacol Ther 2003; 73:275-8.

7. Hogerzeil HV, Walker GJ, Sallami AO, Fernando G. Impact of an essential drugs programme on availability and rational use of drugs. Lancet 1989; 1:141-2.

8. Segovia C, Domecq C, Vargas M, Lemus GI. Essential drugs uses at primary health care. Cuad MédSoc (Santiago de Chile) 1996; 37:57-61. 
9. Gerência Técnica de Assistência Farmacêutica, Departamento de Atenção Básica, Secretaria de Políticas de Saúde, Ministério da Saúde. Relação nacional de medicamentos essenciais - RENAME. 3a Ed. Brasília: Ministério da Saúde; 2002.

10. Organização Pan-Americana da Saúde/Ministério da Saúde. Avaliação da assistência farmacêutica no Brasil: estrutura, processo e resultados. Brasília: Organização Pan-Americana da Saúde; 2005.

11. Santos V, Nitrini SM. Indicadores do uso de medicamentos prescritos e de assistência ao paciente de serviços de saúde. Rev Saúde Pública 2004; 38:819-26.

12. Cunha MCN, Zorzatto JR, Castro LLC. Avaliação do uso de medicamentos na rede pública municipal de saúde de Campo Grande, MS. RBCF Rev Bras Ciênc Farm 2002; 38:215-27.

13. Bontempo VL, Coelho MC, Chaves JC, Pereira LAM, Andrade WW, Braccini CM. Análise das prescrições geradas pelas equipes do PSF e das unidades de atendimento imediato, em dois distritos sanitários do município de Contagem, MG. Bol Pneumol Sanit 2003; 11:17-24.

14. Naves JOS, Silver LD. Evaluation of pharmaceutical assistance in public primary care in Brasília, Brazil. Rev Saúde Pública 2005; 39:223-30.

15. Farias AD, Cardoso MAA, Medeiros ACD, Belém LF, Simões MOS. Indicadores de prescrição médica nas unidades básicas de saúde da família no município de Campina Grande, PB. Rev Bras Epidemiol 2007; 10:149-56.

16. Chaves GC, Emmerick ICM, Pouvourville N, SaintDenis T, Fonseca ASA, Luiza VL. Indicadores de uso racional de medicamentos e acesso a medicamentos: um estudo de caso. Rev Bras Farm 2005; 86 : 97-103.

17. Girotto E, Silva P. A prescrição de medicamentos em um município do norte do Paraná. Rev Bras Epidemiol 2006; 9:226-34.

18. Hogerzeil HV, Bimo, Ross-Degnan D, Laing RO, Ofori-Adjei D, Santoso B, et al. Field tests for rational drug use in twelve developing countries. Lancet 1993; 342:1408-10.

19. Desta Z, Abula T, Beyene L, Fantahun M, Yohannes AG, Ayalew S. Assessment of rational drug use and prescribing in primary health care facilities in north west Ethiopia. East Afr Med J 1997; 74: 758-63.
20. Massele AY, Nsimba SED, Rimoy G. Prescribing habits in church- owned primary health care facilities in Dar-Es-Salaam and other Tanzanian coast Regions. East Afr Med J 2001; 78:510-4.

21. Krause G, Borchert M, Benzler J, Heinmüller R, Kaba I, Savadogo M, et al. Rationality of drug prescriptions in rural health centres in Burkina Faso. Health Policy Plan 1999; 14:291-8.

22. Chareonkul C, Khun VL, Boonshuyar C. Rational drug use in Cambodia: study of three pilot health centers in Kampong Thom Province. Southeast Asian J Trop Med Public Health 2002; 33:418-24.

23. Rehan HS, Lal P. Drug prescribing pattern of interns at a government healthcare centre in northern India. Trop Doct 2002; 32:4-7.

24. Mahfouz AA, Shehata AI, Mandil AM, Al-Erian RA, Al-Khuzaeyem AA, Kisha A. Prescribing patterns a primary health care level in the Asir Region, Saudi Arabia: an epidemiologic study. Pharmacoepidemiol Drug Saf 1997; 6:197-201.

25. Trevisol DJ. Influência da propaganda da indústria farmacêutica na prescrição de medicamentos em escolas de medicina: a experiência em Tubarão, Santa Catarina [Dissertação de Mestrado]. Tubarão: Universidade do Sul de Santa Catarina; 2006.

26. Laing RO, Hogerzeil HV, Ross-Degnan D. Ten recommendations to improve use of medicines in developing countries. Health Policy Plan 2001; 16: 13-20.

27. Ministério da Saúde. Portaria GM no. 3.916, de 30 de outubro de 1998. Aprova a Política Nacional de Medicamentos. http://portal.saude.gov.br/portal/ arquivos/pdf/portaria3916_30out98.pdf (acessado em 10/Out/2009).

28. Conselho Nacional de Saúde. Resolução nº. 338, de 6 de maio de 2004. Aprova a Política Nacional de Assistência Farmacêutica. http://portal.saude.gov. br/portal/arquivos/pdf/resolucao_338_politica_ ass_farmaceutica.pdf (acessado em 10/Out/2009).

29. World Health Organization. How to investigate drug use in health facilities. Geneva: World Health Organization; 1993.

30. World Health Organization Collaborating Centre for Drug Statistics Methodology. Anatomical Therapeutic Chemical (ATC) index with defined daily doses (DDDs). http://www.whocc.no/atc_ddd_in $\mathrm{dex} /$ (acessado em 21/Dez/2009).

Recebido em 30/Abr/2009

Versão final reapresentada em 22/Dez/2009

Aprovado em 02/Fev/2010 